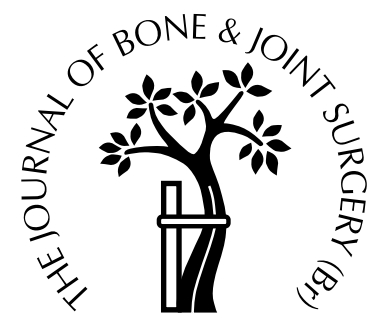

\title{
Iliosacral screw fixation of the posterior pelvic ring using local anaesthesia and computerised tomography
}

\author{
B. H. Ziran, W. R. Smith, J. Towers, S. J. Morgan \\ From the Universities of Pittsburgh and Colorado, USA
}

$V^{2}$ arious techniques have been used for the fixation of the posterior pelvis, each with disadvantages specific to the technique. In this study, a new protocol involving the placement of posterior pelvic screws in the CT suite is described and evaluated.

A total of 66 patients with unstable pelvic ring injuries was stabilised under local anaesthesia with sedation. The mean length of time for the procedure was 26 minutes per screw. There were no technical difficulties or misplaced screws and no cases of infection or nonunion. All patients stated that they would choose to have the CT scan procedure again rather than a procedure requiring general anaesthesia. The charges for the procedure were approximately $£ 1840$ (\$2800) per operation. CT-guided placement of iliosacral screws is a safe, feasible, and cost-effective alternative to radiologically-guided placement in the operating theatre in selected patients.

J Bone Joint Surg [Br] 2003; 85-B:411-8. Received 4 January 2002; Accepted after revision 31 July 2002

Percutaneous iliosacral screws are commonly used for the fixation of the posterior pelvis. Several authors have described the technique in detail and reported excellent results. ${ }^{1-5}$ The procedure is technically demanding because of the limitations of radiological visualisation of the relevant landmarks. The complex anatomy and the presence of sacral dysmorphism may make it difficult to place the

B. H. Ziran, MD, Assistant Professor

Department of Orthopaedic Surgery

J. Towers, MD, Assistant Professor

Department of Radiology

University of Pittsburgh, 3471 5th Avenue, Suite 1010, Pittsburgh, Pennsylvania 15213 , USA.

W. R. Smith, MD, Assistant Professor

S. J. Morgan, MD, Assistant Professor

Department of Orthopaedic Surgery, University of Colorado Health Sciences Centre, Denver Health Medical Centre, Denver, Colorado 80204, USA.

Correspondence should be sent to Dr B. H. Ziran.

(C)2003 British Editorial Society of Bone and Joint Surgery doi.10.1302/0301-620X.85B3.13119\$2.00

\begin{tabular}{|c|c|}
\hline Classification & $\begin{array}{l}\text { Number of } \\
\text { patients }\end{array}$ \\
\hline \multicolumn{2}{|l|}{ Young-Burgess } \\
\hline APC2 & 11 \\
\hline APC3 & 17 \\
\hline $\mathrm{LC} 2$ & 14 \\
\hline LC3 & 6 \\
\hline VS & 18 \\
\hline \multicolumn{2}{|l|}{$\mathrm{AO}$} \\
\hline C1 & 60 \\
\hline $\mathrm{C} 3$ & 6 \\
\hline
\end{tabular}

screws accurately under radiological control. ${ }^{6-12}$ There have been reports of misplaced screws and other complications, occasionally with serious consequences. ${ }^{2}$ Efforts to improve the accuracy of placement have included using open techniques and CT guidance. The open technique provides only limited access to the sacroiliac (SI) junction (anterior or posterior) and requires a significant surgical approach with its associated morbidities. In order to place iliosacral screws under CT control, anaesthetists, anaesthetic equipment, and operating-theatre personnel should be taken to the radiology department, with draping and technique similar to that used in the operating theatre. ${ }^{13-17}$ We have developed a protocol for introducing the screws in the CT suite, using local anaesthesia and sedation, with a minimum of equipment and personnel. The potential advantages include ease and accuracy of placement of the screw, the ability to monitor the neurological status in a conscious patient, and reduced cost. We now evaluate this technique in a consecutive series of patients.

\section{Patients and Methods}

Between July 1996 and July 1999, 550 patients with pelvic fractures were treated at our institutions, 330 non-operatively and 220 with stabilisation. Using our protocol for treating disruptions of the posterior pelvic ring, 66 met the inclusion criteria and were stabilised using the CT-guided technique. Informed consent was obtained from the patient 


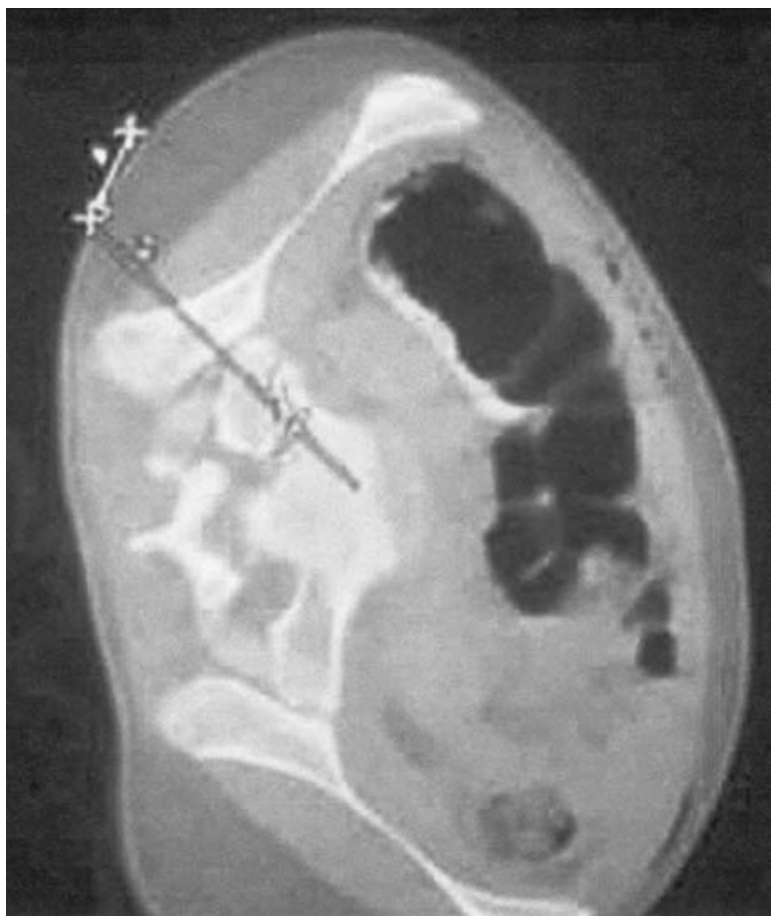

Fig. 1

CT scan showing that using the monitor image, the desired trajectory of the screw can be drawn. The cutaneous projection of this trajectory is then measured to the radiopaque marker. This corresponding length is marked with an indelible pen mark along the transverse laser sight.

or an immediate family member. There were 38 men and 28 women with a mean age of 42 years (12 to 78). The mean follow-up was 25 months (18 to 42). The mechanism of injury was a motor-vehicle or motor-cycle accident in 55, a fall in ten and a ski-ing accident in one. There were other injuries in $43 \%$ of the patients. The fractures were classified by both the Young-Burgess and the AO systems (Table I). The injuries were evenly distributed between left and right sides, and there were six bilateral injuries.

A prospective protocol was designed for the placement of the iliosacral screws, using local anaesthesia and sedation in the radiology suite. The indications for this technique were unstable pelvic ring injuries ( $\mathrm{AO}$ types $\mathrm{B}$ and $\mathrm{C}$ ), for which iliosacral screw fixation was chosen for posterior fixation. All patients were haemodynamically stable at the time of the procedure. Exclusion criteria included those who were haemodynamically unstable and those with thoracic or abdominal wounds which prevented semilateral positioning. Also excluded were those in whom iliosacral screw placement was part of another procedure, such as the open reduction and internal fixation of a crescent fracture, or tensionband plating of displaced bilateral iliosacral fracture dislocations. For intubated patients, the ventilator and intensivecare nurse were brought to the radiology suite, and the patients were monitored as for any procedure which is carried out in the intensive-care unit.

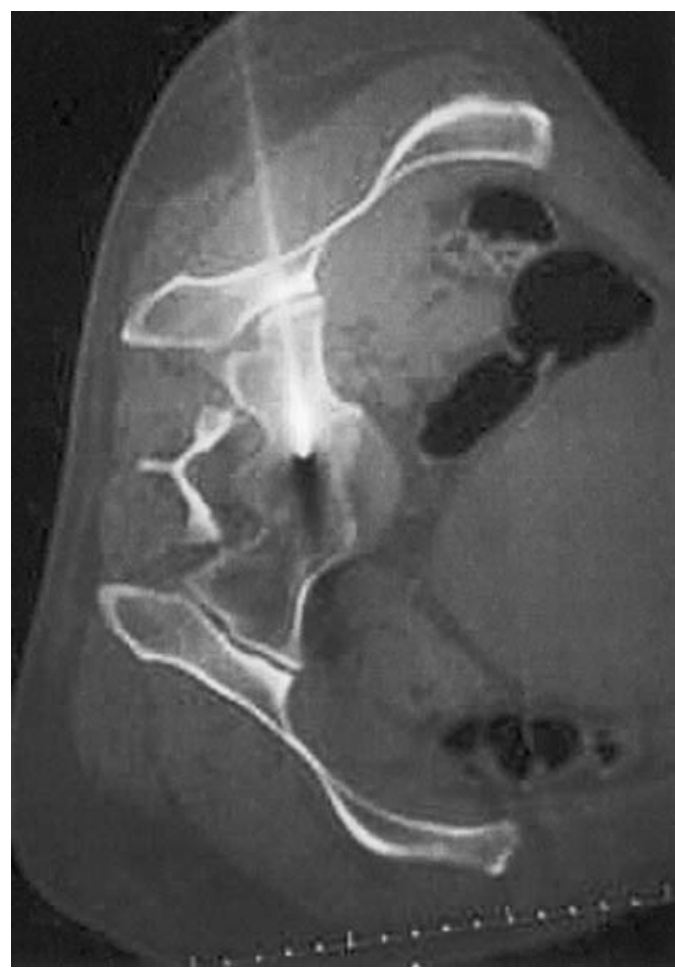

Fig. 2

CT scan showing the guide-wire within the sacral body. The depth of penetration and the length of the screw can be directly measured from $\mathrm{CT}$ monitor software.

Minimally displaced fractures were fixed in situ if they were considered to be unstable or if they were associated with significant symptoms. If the fracture appeared to be undisplaced on radiographs and CT, those with significant symptoms and with comminuted multiple ring injuries such as a comminuted posterior and bilateral ramus fracture, were considered to be radiologically stable, but clinically unstable, and were included. Some patients with displaced fractures were treated by initially stabilising the anterior lesion by internal or external fixation. The posterior lesion was assessed again and, if satisfactorily reduced, was stabilised in situ by the CT-guided procedure. In such patients our methodology was based on the established protocol with attempts to avoid open posterior techniques or radiologically placed posterior screws. Thus, instead of having both an anterior open procedure and a posterior open procedure and/or screw, we first addressed the anterior lesion, usually on the day of presentation, and then assessed the appropriateness of using the CT-guided technique for the posterior lesion. Occasionally, a closed reduction was attempted with the use of traction or by manipulation with an external fixator in the radiology suite. In several fractures which could not be adequately reduced, 'reduction screws' were used. These were placed in order to reduce the displaced fragment and as they tightened, the fracture would reduce to an acceptable position and iliosac- 


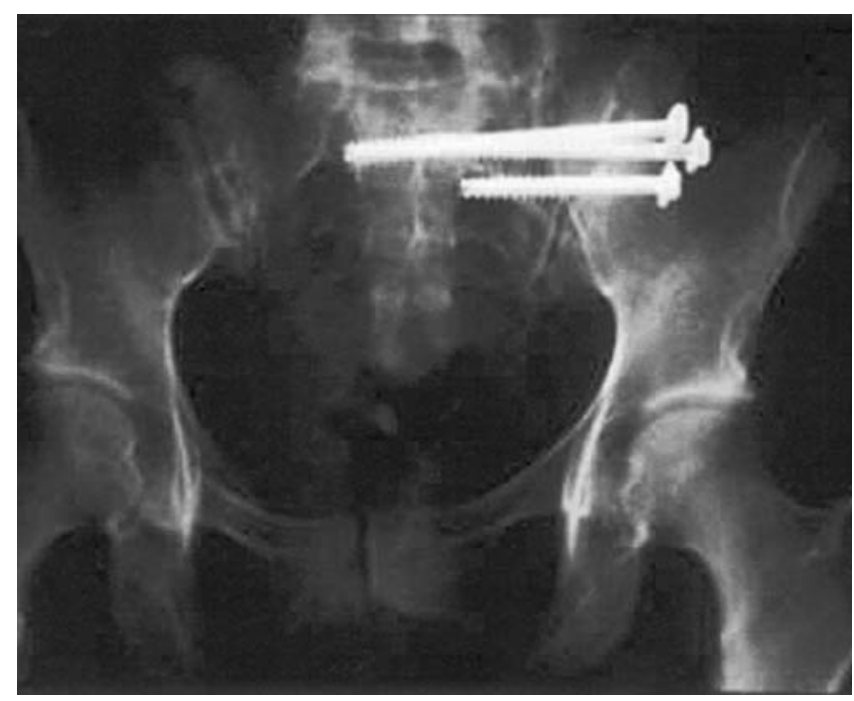

Fig. 3

Corresponding anteroposterior (AP) pelvic radiograph of Figure 7.

ral screws could be placed for stabilisation. If the reduction was not adequate or if the patients could not tolerate these manoeuvres, the fixation was undertaken in the operating room.

In addition to routine clinical details, we recorded the total time for the procedure, including time per screw, the total radiation dosage, the amount of local anaesthesia and sedation used, complications such as infection, technical failure, or logistical problems, the adequacy of reduction of the fracture and its healing, and the accuracy of placement of the screws. We also evaluated how the patients tolerated the procedure using a subjective pain scale (range 0 to 10). A cost analysis was undertaken by comparing the cost of the CT-guided procedure with that of isolated iliosacral screw placement carried out in the operating theatre. Only isolated single screw placements were reviewed in the cost analysis which included the time in the operating theatre, the anaesthetic and medication costs, and the time in the recovery room.

Operative technique. Patients were placed and supported in a semilateral position on the scanner bed. Intravenous antibiotics were administered and local antisepsis and sterile draping used. The scanner bed was on a calibrated track so that the same images could be used and repeated throughout the procedure. Between three and six images (5 $\mathrm{mm}$ slice thickness) were obtained around the desired position of the screw, which was usually in the pedicle of $\mathrm{S} 1$ or S2. The cutaneous site of insertion was localised relative to a radiopaque marker on the skin. The laser sights of the CT gantry indicated the cutaneous location of the CT image slice. The transverse laser sight marked the cutaneous site which corresponded to the underlying osseous level. Using the measuring software, the distance from the marker to the proposed line of trajectory was measured on the monitor.
The distance from the marker on the skin along the laser sight was measured and the exact site of entry of the screw determined (Fig. 1).

The patient was then withdrawn from the CT gantry, prepared and draped. Intravenous sedation was administered using fentanyl (50 $\mu \mathrm{g}$ increments) and Versed $(0.5 \mathrm{mg}$ increments). Local anaesthesia consisting of a mixture of $0.5 \%$ bupivicaine and $1 \%$ lidocaine was administered to the skin, subcutaneous tissues, and along the outer iliac periosteal surface. A biopsy trephine was used to open the external iliac cortex and advanced to the subchondral bone of the SI joint. Local anaesthetic was intermittently administered through a cannula into the trajectory of the trephine into the SI pedicle. Before advancing the trephine across the SI joint, the CT images were repeated to confirm its position. The length of the screws which were required was established using the measuring software provided with the scanner. More local anaesthetic was administered through the cannula of the trephine. Using battery-powered equipment (Stryker, Kalamazoo, Michigan), a guide-wire for a $7.3 \mathrm{~mm}$ self-drilling and tapping cancellous screw (Synthes, Paoli, Pennsylvania) was placed through the trephine into the sacral vertebral body (Fig. 2). The appropriate-sized screw and washer were then inserted. This was the most painful part of the procedure. When all the screws were in place, the final reduction was checked on CT and radiographs (Fig. 3) before the small stab wounds were closed.

If the reduction of the posterior pelvis was not accurate, several manoeuvres were attempted. First, if there was an external fixator on the pelvis, it was manipulated to effect a better reduction. A repeat scan confirmed the reduction. If there was only a slight residual displacement, reduction screws were used to improve the reduction. The direction of the desired reduction was determined and a proposed trajectory chosen. The reduction screw was placed in order to reduce the pelvis and allow the placement of standard screws to compress the SI joint. Figures 4 to 9 show the sequence of images during the use of a reduction screw.

\section{Results}

A total of 113 screws was introduced into the 66 patients; 80 into S1, 31 into S2 and two into S3. The mean time for the procedure was 26 minutes per screw (18 to 45$)$. The mean level of pain was five out of ten (0 to 9 ). The maximum pain occurred during insertion of the screws. The mean amount of sedation was $142 \mu \mathrm{g}$ (0 to 450) of fentanyl and $3.0 \mathrm{mg}$ ( 0 to 5.0) of Versed per screw. The mean amount of local anaesthesia used was $19 \mathrm{ml}$ ( 8 to 40) of lidocaine and $11 \mathrm{ml}$ (5 to 16) of bupivicaine per screw.

In 13 cases of anterior symphyseal plating and in three fractures, external fixation was used before placement of the iliosacral screw. In all but five of these the posterior reduction after anterior fixation was considered to be nearly anatomical and fixation was undertaken in situ. In another fxive 


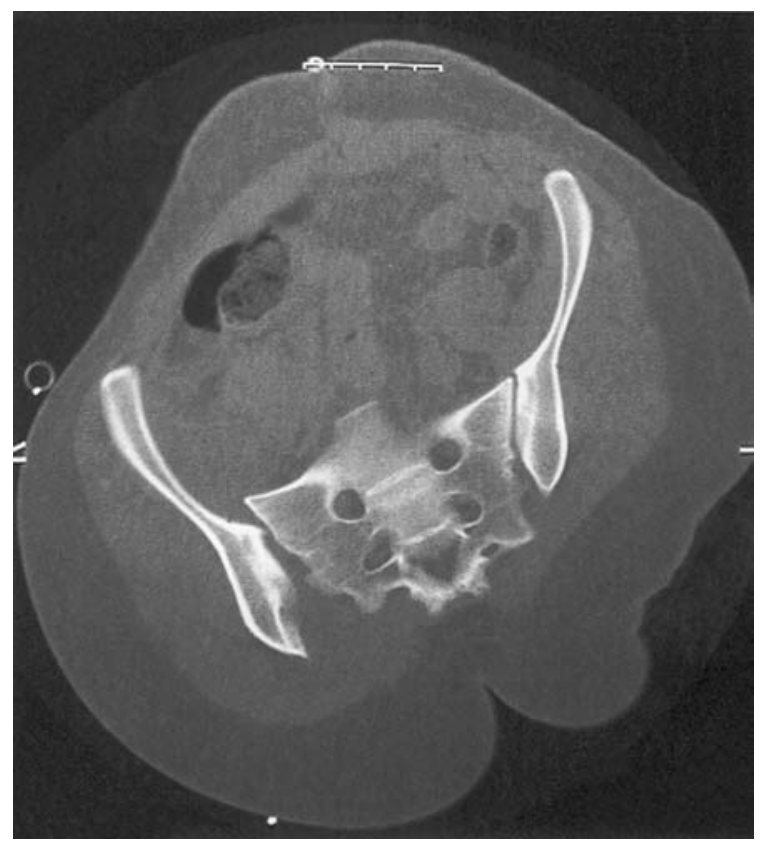

Fig. 4

CT scan of a widely displaced SI dislocation. This patient, with an AO type C1.2 injury, also had an associated pubic symphyseal disruption. Although there is posterior displacement and internal rotation of the ilium, the cephalocaudal positioning was considered to be satisfactory.

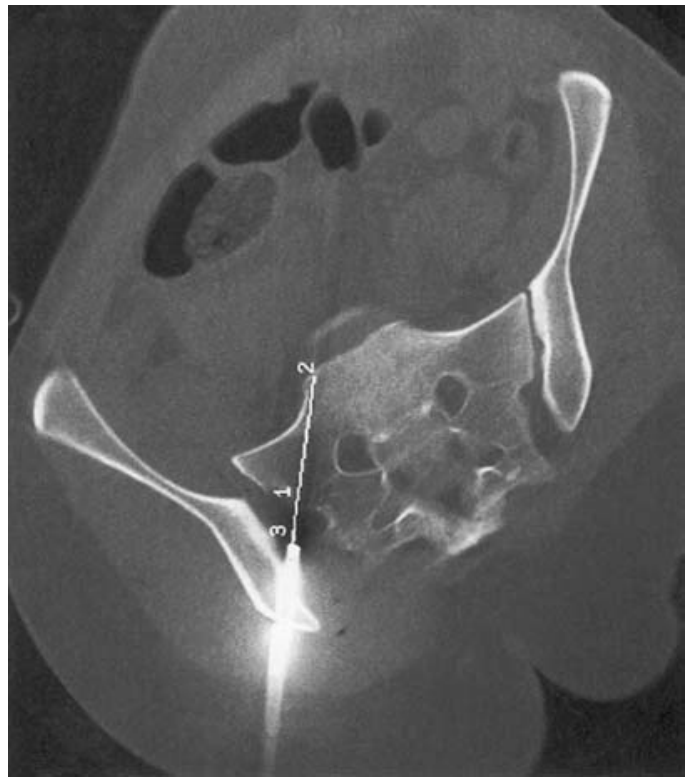

Fig. 6

CT scan of the position of the trephine and the extrapolated trajectory of the proposed reduction screw. When choosing the length of the screw, the distance of reduction must be accounted for, so that the screw does not protrude anterior to the sacral body.

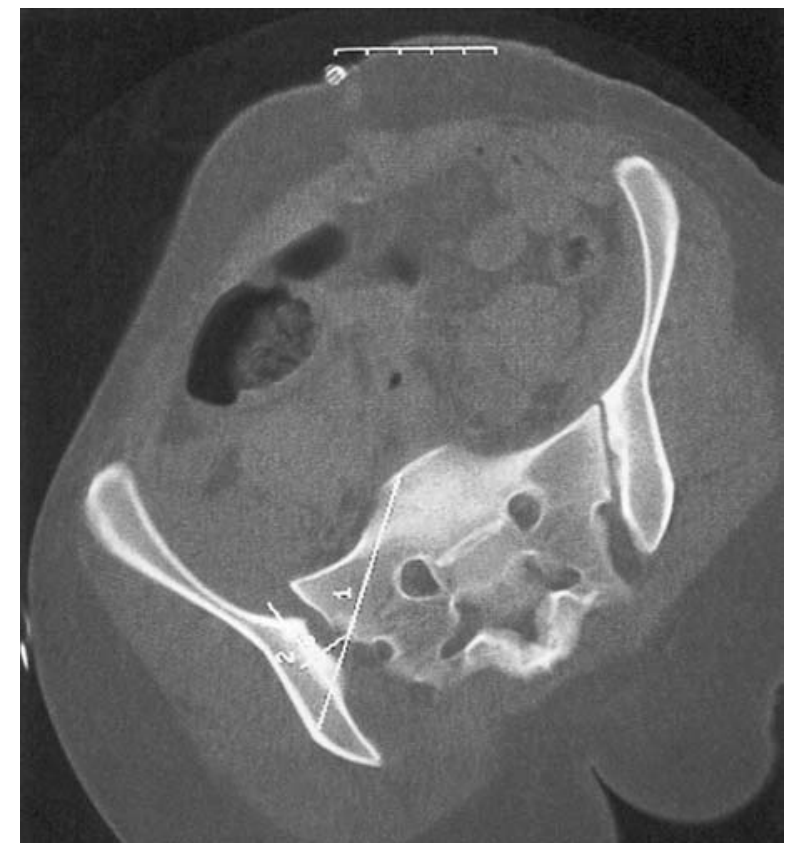

Fig. 5

CT scan showing that to effect a reduction, anterior translation of the ilium was required. Using CT software, the proposed trajectory of the reduction screw was drawn. The technique for cutaneous location showed that a more posterior entry site was required.

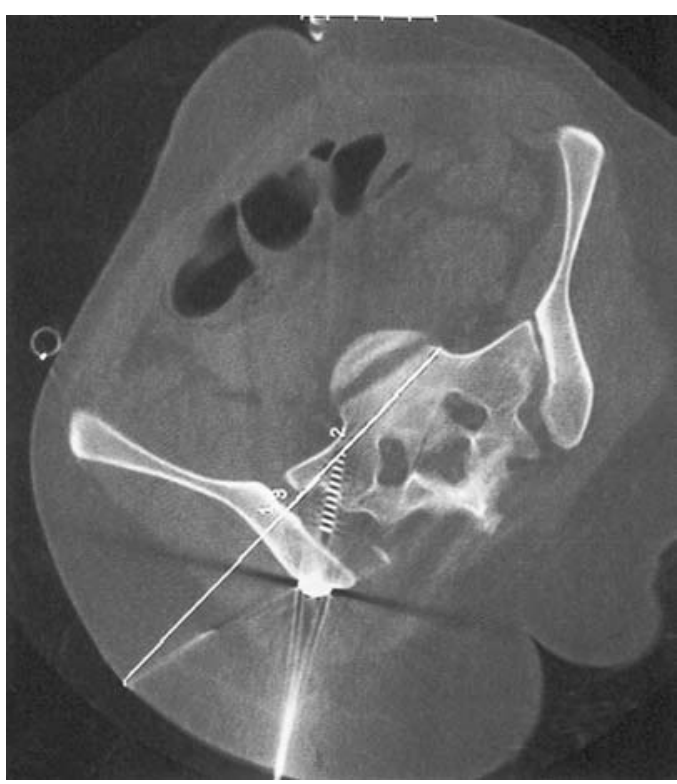

Fig. 7

CT scan showing the anterior reduction obtained with the reduction screw. There is still a residual diastasis of the SI joint. This is fixed with a standard SI screw with the proposed trajectory of which is drawn on the CT monitor image. 


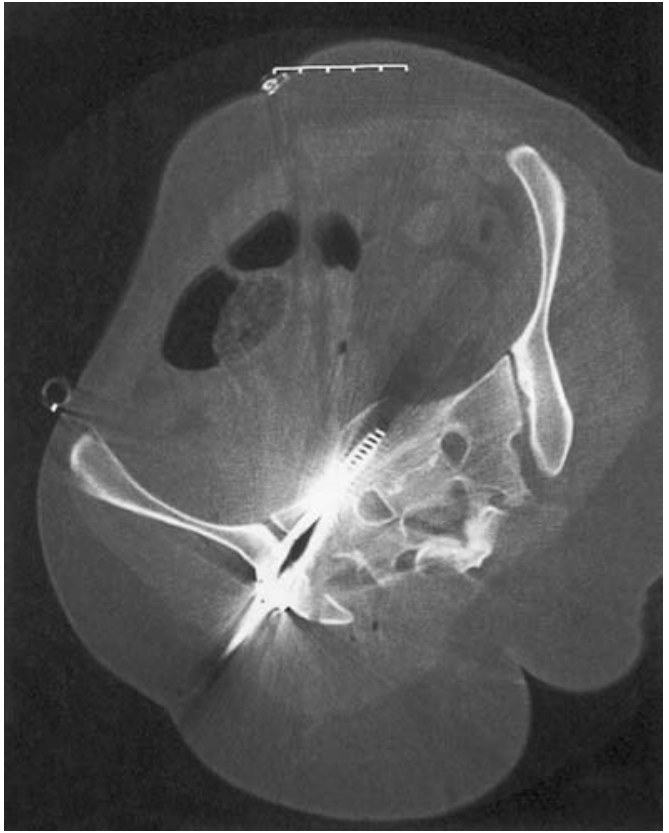

Fig. 8

CT scan showing that the final seating of the standard SI screw gives reduction of the residual diastasis with maintenance of the cephalocaudal reduction. To perform this final reduction, the original screw must be removed. This should be done only after the standard screw has crossed the gap and engaged into the $\mathrm{S} 1$ body. Removal of the reduction screw before this risks loss of reduction.

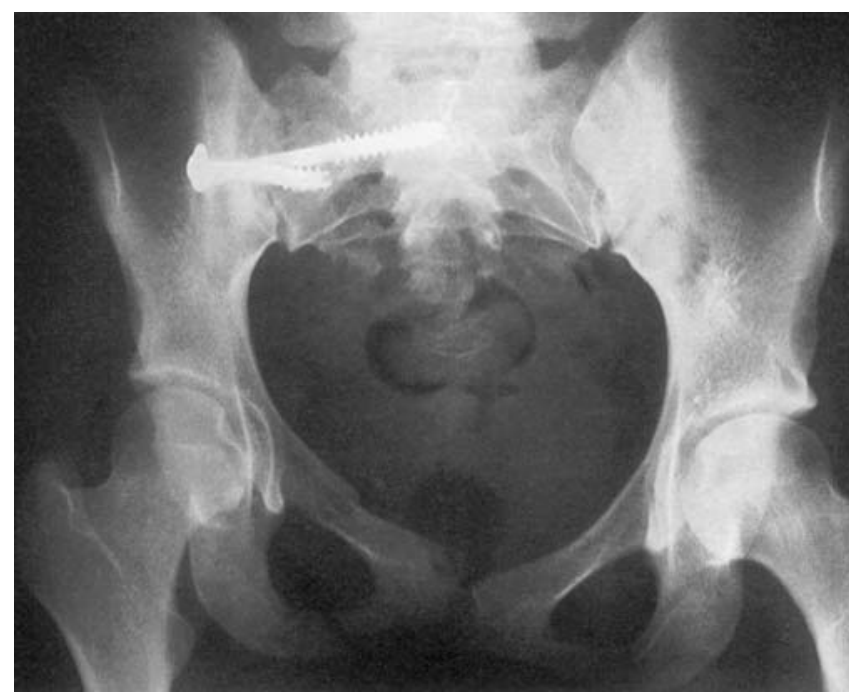

Fig. 9

Postoperative AP radiograph showing reduction and the position of the screws.

(three with external fixation and two with plating), closed reduction with reduction screws was carried out. The final reductions were considered to be satisfactory and these patients tolerated the procedure with a mean maximum pain score of 6.25 out of 10 . None of these required formal inter- nal fixation because of an inability to achieve a satisfactory reduction.

There were no technical difficulties, logistical problems, or misplaced screws. One patient required mask anaesthesia to complete the procedure. There were no cases of infection or nonunion. One non-compliant patient with a $\mathrm{C} 1$ fracture who underwent internal fixation and two posterior screws, began immediate weight-bearing and had a healed fracture with a displacement of $5 \mathrm{~mm}$ and a broken screw at final follow-up, but was asymptomatic. Most patients had only a vague recollection of the procedure and were pleased with the outcome and all stated that they would choose to have the CT scan procedure again rather than a procedure requiring general anaesthesia.

Analysis of cost compared only procedural and equipment cost not shared between the operating theatre and CTguided procedures. The charges for the procedure, including CT usage, equipment, radiology, and nursing fees approximated $£ 1840$ (\$2800) per operation. The surgeon’s fee was constant independent of the setting and was not included. Charges for operative placement with radiology ranged from $£ 4934$ to $£ 7895$ (\$7500 to $\$ 12000$ ).

\section{Discussion}

Pelvic fractures which require operative stabilisation remain a challenge to the orthopaedic surgeon because of the technical difficulty. The use of iliosacral screw fixation for some posterior lesions is an elegant method of stabilisation, but complications may arise because of poor visualisation or understanding of the posterior pelvic anatomy. Routt et $\mathrm{al}^{2}$ reported 177 patients and found imaging problems in 10\% because of body habitus, bowel gas, or inadequate machinery. They also described five examples of malpositioned screws. In another study, there was an incidence of $13 \%$ of malpositioned screws, although with no sequelae. ${ }^{18}$ Damage to the superior gluteal artery has been reported. ${ }^{19}$ Templeman et $\mathrm{al}^{20}$ showed that malposition of the screw by as little as $4^{\circ}$ could cause damage to neurovascular structures. The technical problems of screw placement are compounded by difficulties of radiological interpretation and variations in the anatomy of the posterior pelvis. ${ }^{21}$ Routt et $\mathrm{al}^{4}$ emphasised the use of the lateral image and the sacral alar slope and iliac cortical density to establish the 'safe zone'. Other CT studies of the alar root show that the bottleneck of this safe corridor of bone is the posterior-medialcephalad area. ${ }^{9}$

Unfortunately, many of the borders of the volume of bone available for screw placement are not well seen radiologically. Ziran et $\mathrm{al}^{22}$ found that even under ideal conditions, the cephalad, caudal, and posterior borders were difficult to visualise. The alar slope and anterior alar cortices were the only practical and reproducible landmarks available. Thus, variations in alar morphology and the course of the neural foramen could cause problems with the imaging and placement of devices. One possible solution was pro- 
posed by Moed et $\mathrm{al}^{23}$ and Webb et $\mathrm{al}^{24}$ who used intraoperative monitoring with evoked potentials and electromyography. They found that redirection was required in about $8 \%$ of operations. Although they avoided neurological injury, the fundamental problem remained that of accurate imaging and visualisation during the procedure, and of identifying vascular dangers. All these difficulties can be circumvented by the use of CT guidance. In our series, all screws were shown to be extraforaminal and within sacral bone. It was also much easier to ensure appropriate orientation of screws in a plane perpendicular either to the sacral fracture or the SI joint.

CT-guided placement of iliosacral screws was described by Nelson and Duwelius, ${ }^{16}$ and later by Duwelius et $\mathrm{al}^{25}$ and Ebraheim et al. ${ }^{13}$ Using general anaesthesia and an arrangement similar to that in the operating theatre, they reported good results, but were unable to carry out reductions of displaced fractures and noted logistical difficulties with regard to draping, operating equipment, and anaesthetic requirements. Our series differs from previous reports in that we used only local anaesthesia and sedation and did not turn the radiology suite into an operating theatre. We only used single-layer, block draping and an aseptic technique. Initially, we had difficulty in maintaining the sterility of the guide-wire as it entered the gantry during successive scans. Cutting it with a bolt cutter allows safe passage without contamination. There is the potential for contamination from the CT gantry, but we have had no instances of infection to date. When a patient from the surgical intensive-care unit was already intubated, normal cardiopulmonary monitoring was undertaken by a critical care nurse, as would occur for any procedure in the intensive-care unit. No anaesthetists were required, but close monitoring with a pulse oximeter, vital signs, and verbal interactions with the patient are recommended. The amount of sedation and local anaesthesia was relatively constant, and the pain scale scores were acceptable.

In the early stages of the study, only patients with disruptions of the pelvic ring which were considered biomechanically unstable, but did not require a formal open reduction of the posterior pelvis, underwent CT-guided iliosacral screw placement. For example, patients with significant anterior injuries with comminuted, but minimally displaced posterior lesions, were felt to have unstable lesions with a high risk of displacement, and thus were stabilised by posterior iliosacral screw fixation. These patients also had significant clinical symptoms. Thereafter, we also included patients with displaced anterior and posterior injuries, in whom reduction and fixation of the anterior ring resulted in a satisfactory posterior reduction. These patients were first stabilised in the operating theatre and then the posterior lesions were stabilised by CT guidance. Later in the study, small or moderate posterior displacements were corrected with a closed reduction using manipulation of an external fixator and/or reduction screws. As our experience grew, we began to undertake closed reductions and were successful in

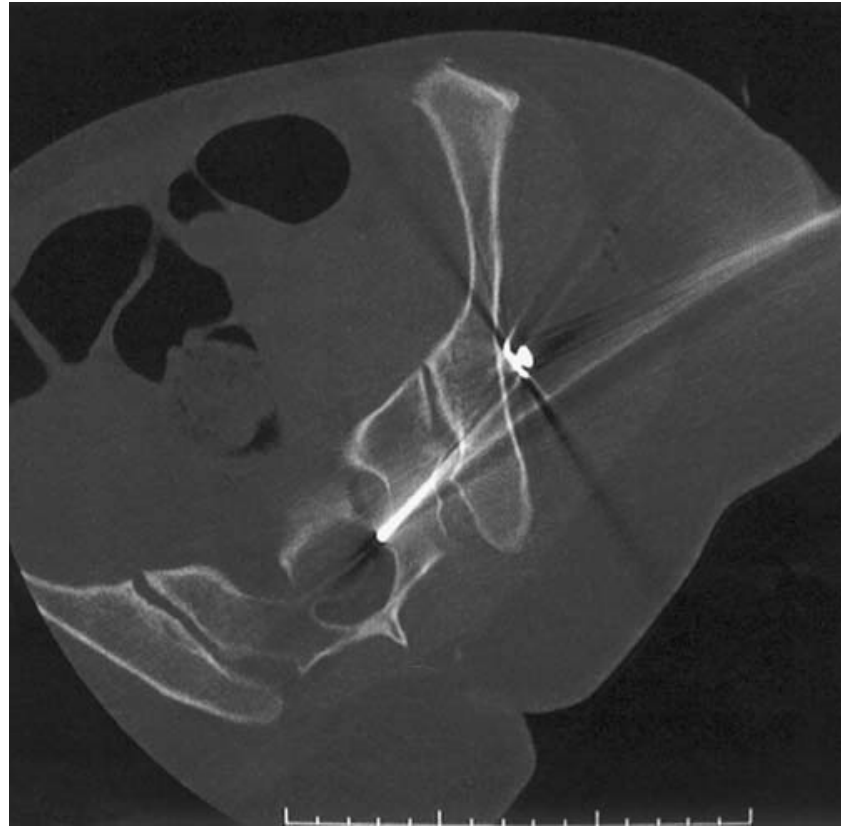

Fig. 10

CT scan showing the placement of a screw posterior to the foramen in a location not possible using standard radiological techniques. This and other locations can be accessed by the CT-guided technique.

six patients. We caution against the indiscriminate use of this technique, because repeated manipulations of the pelvis are not only uncomfortable in a conscious patient, but could dislodge a stable haematoma or, in transforaminal fractures, result in neurological injury. An awake patient is a distinct advantage in the latter situation.

In some fracture patterns, notably comminuted sacral fractures and sacral fractures in osteopenic bone, it may be desirable to place a second iliosacral screw in either S1 or $\mathrm{S} 2$. The first screw is often placed in S1 without difficulty, but the sacral anatomy or the limitations of radiological imaging make placement of a second screw in an appropriate site difficult. This occurred in several of our patients, and they were subsequently taken to the radiology suite where both an S1 screw and a second screw, usually in S2, were placed accurately and without difficulty. One other advantage of this technique is the opportunity to place screws in positions not accessible by standard radiological visualisation. For example, Figure 10 shows a screw placed posterior to the foramen and anterior to the spinal canal. This position in the posterior pelvis cannot be visualised well enough by standard radiological methods to allow safe placement. The ability to access all the available osseous areas of the posterior pelvis and sacrum is especially helpful in certain fracture patterns or in dysmorphic pelvises.

Sacral dysmorphism is present in an undetermined, but significant percentage of the normal population. In our series, all patients with sacralised L5 vertebral bodies had altered sacral anatomy, including eccentrically placed S1 
and S2 foramina and narrow or absent S1 pedicles and body. In these patients, attempted placement of an iliosacral screw using radiological visualisation carried a risk of misplacement of a screw into a foramen or into the central canal of the sacrum. CT guidance, in these patients, allowed for precise placement of $7.3 \mathrm{~mm}$ screws, often through very narrow (10 to $14 \mathrm{~mm}$ ) corridors. Open reduction techniques would not have provided any advantage for safe placement of screws because the intrasacral anatomy and the positions of the foramina would still not have been visualised. Direct posterior plating from one iliac wing to another may be an alternative, but this technique is biomechanically less stable and requires extensive dissection and potential morbidity.

We found a distinct advantage of CT-guided placement of iliosacral screws in several other groups of patients. The difficulty of visualising appropriate landmarks radiologically in obese patients, those with excessive bowel gas, and in children has been noted by previous authors. ${ }^{2,22}$ The CTguided technique allows for complete visualisation and safe passage of screws at the appropriate angle for an effective reduction.

The advent of computer-assisted orthopaedic surgical (CAOS) techniques now allows a computer model of the patient to be created through a method of registration of anatomical landmarks. ${ }^{7,26}$ Using such techniques, both radiological and CT guidance may possibly be avoided, thus greatly facilitating such procedures. The accuracy of such products to within 1 to $2 \mathrm{~mm}$ makes them very desirable in trauma and in particular for such complex three-dimensional structures as the pelvis. Most of the preliminary work using CAOS has been in the spine and with arthroplasty. Although clearly useful, these products are exceedingly expensive and we did not have them at our institution. As such equipment becomes more readily available and affordable, it would potentially preclude the need for radiologically or CT-guided techniques.

As part of our learning curve, we found that the time needed for the insertion of screws and the amount of sedation which was required decreased. The critical judgement of the procedure is determining the correct indication and trajectories. Once the logistics of the scanner, technologists, and radiologists are negotiated, the operation proceeds rather simply. The use of fluoroscopic CT has recently become available at our institution and this can shorten the procedure time even further. We do not advocate the CT technique as a replacement for standard radiological techniques, but as a complement. The technique will be most applicable for surgeons who are not proficient with radiologically placed SI screws, or for those who do not have appropriate operative equipment and staff. It allows for a safe and effective method of stabilising the posterior pelvis. We have also used the technique as a method of sacroiliac stabilisation for chronic sacroiliac disorders.

In a select group of patients, who require pelvic stabilisation using iliosacral screws, but do not require open reduc- tion, percutaneous placement with $\mathrm{CT}$ guidance and portable equipment is feasible. It should not be used if the posterior reduction is unacceptable and open reduction is required, when more traditional methods should be used. Although we undertook closed reduction of the fracture in the radiology suite, understanding the true three-dimensional problem using two-dimensional CT images can be very difficult. There should be a low threshold to abort the procedure and pursue treatment in the operating theatre. When open reduction and internal fixation is required, the screw can be placed at the same time and a separate trip to the scanner is not indicated. Additionally, if there are not sufficient resources available (nurses, anaesthetist on call, etc) for the procedure, which would include resuscitation if needed, then using the operating theatre would still be preferable. CT-guided placement of iliosacral screws is a safe, feasible, and cost-effective alternative to radiologicallyguided placement in the operating theatre in selected patients.

No benefits have been received or will be received from a commercial party related directly or indirectly to the subject of this article.

\section{References}

1. Matta JM, Saucedo T. Internal fixation of the pelvic ring fractures. Clin Orthop 1989;242:83-97.

2. Routt ML Jr, Simonian PT, Mills WJ. Iliosacral screw fixation: early complications of the percutaneous technique. J Orthop Trauma 1997;11:584-9.

3. Routt ML Jr, Kregor PJ, Simonian PT, Mayo KA. Early results of percutaneous iliosacral screws placed with the patient in the supine position. J Orthop Trauma 1995;9:207-14.

4. Routt ML Jr, Simonian PT, Agnew SG, Mann FA. Radiographic recognition of the sacral alar slope for optimal placement of iliosacral screws: a cadaveric and clinical study. J Orthop Trauma 1996;10:171-

5. Routt ML Jr, Simonian PT, Inaba RT. Iliosacral screw fixation of the disrupted sacroiliac joint. Tech Orthop 1995;9:300.

6. Amongero ME, Wilber JH. Upper sacral morphology and its relation to sacroiliac screw fixation. Orthop Trans 1995;19:435.

7. Barrick EF, O'Mara JW, Lane HE. Iliosacral screw insertion using computer-assisted CT image guidance: a laboratory study. Comput Aided Surg 1998;3:289-96.

8. Carlson DA, Scheid DK, Maar DC, Baele JR, Kaehr DM. Safe placement of S1 and S2 iliosacral screws: the "Vestibule" concept. J Orthop Trauma 2000;14:264-9.

9. Day CS, Prayson MJ, Shuler TE, Towers J, Gruen GS. Trans-sacral versus modified pelvic landmarks for percutaneous iliosacral screw placement: a computed tomographic analysis and cadaveric study. Am J Orthop 2000;29(Suppl):16-21.

10. Ebraheim NA, Xu R, Biyani A, Nadaud MC. Morphologic considerations of the first sacral pedicle for iliosacral screw placement. Spine 1997;22:841-6.

11. Goldberg BA, Lindsey RW, Foglar C, et al. Imaging assessment of sacroiliac screw placement relative to the neuroforamen. Spine 1998;23:585-9.

12. Noojin FK, Malkani AL, Haikal L, Lundquist C, Voor MJ. Crosssectional geometry of the sacral ala for safe insertion of iliosacral lag screws: a computed tomography model. J Orthop Trauma 2000;14:31-5.

13. Ebraheim NA, Coombs R, Jackson WT, Rusin JJ. Percutaneous computed tomography-guided stabilization of posterior pelvic fractures. Clin Orthop 1994;307:222-8.

14. Eude P, Damon F, Eude G, et al. Percutaneous osteosynthesis of pelvic fractures with CT control. J Radiol 2000;81:63-8.

15. Jacob AL, Messmer $P$, Stock KW, et al. Posterior pelvic ring fractures: closed reduction and percutaneous CT-guided sacroiliac screw fixation. Cardiovasc Intervent Radiol 1997;20:285-94. 
16. Nelson DW, Duwelius PJ. CT-guided fixation of sacral fractures and sacroiliac joint disruptions. Radiology 1991;180:527-32.

17. Tonetti J, Carrat L, Lavellee S, et al. Percutaneous iliosacral screw placement using image guided techniques. Clin Orthop 1998;354:10310.

18. Keating JF, Werier J, Blachut $\mathbf{P}$, et al. Early fixation of the vertically unstable pelvis: the role of iliosacral screw fixation of the posterior lesion. J Orthop Trauma 1999;13:107-13.

19. Altman DT, Jones CB, Routt ML Jr. Superior gluteal artery injury during iliosacral screw placement. J Orthop Trauma 1999;13:220-7.

20. Templeman D, Schmidt A, Freese J, Weisman I. Proximity of iliosacral screws to neurovascular structures after internal fixation. Clin Orthop 1996;329:194-8.

21. Ebraheim NA, Haman SP, Xu R, Stenescu S, Yeasting RA. The lumbosacral nerves in relation to dorsal S1 screw placement and their locations on plain radiographs. Orthopedics 2000;23:245-7.
22. Ziran BH, Wason AG, Olsen S, Chapman MW. Radiographic anatomy of the iliosacral corridor. Procs Orthopaedic Trauma Association, 1996:192.

23. Moed BR, Hartman MJ, Ahman BK, Cody DO, Craig JG. Evaluation of intraoperative nerve monitoring during insertion of an iliosacral implant in an animal model. J Bone Joint Surg [Am] 1999;81-A:152937 .

24. Webb LX, de Araujo W, Donofrio P, et al. Electromyography monitoring for percutaneous placement of iliosacral screws. J Orthop Trauma 2000;14:245-54.

25. Duwelius PJ, VanAllen M, Bray TJ, Nelson D. Computed tomography-guided fixation of unstable posterior pelvic ring disruptions. $J$ Orthop Trauma 1992;6:420-6.

26. Gautier E, Bachler R, Heini PF, Nolte LP. Accuracy of computerguided screw fixation of the sacro-iliac joint. Clin Orthop 2001;393:310-7. 